\title{
Neuropsychiatric Systemic Lupus Erythematosus: Making the Case for an Expanded Psychiatric Role
}

\author{
Ayol Samuels", Mirnova E. Ceïde and Naalla Schreiber \\ Department of Psychiatry \& Behavioral Sciences, Montefore Medical Center/Albert Einstein College of Medicine, New York, USA
}

Correspondence to:

Dr. Ayol Samuels, MD

Department of Psychiatry

Montefiore Medical Center/Albert Einstein

College of Medicine, 111 East 210th street

Bronx, NY 10467, USA

Tel: 9177512671

E-mail: ayol.samuels@gmail.com

Received: March 09, 2016

Accepted: June 15, 2016

Published: June 17, 2016

Citation: Samuels A, Ceïde ME, Schreiber N.2016. Neuropsychiatric Systemic Lupus Erythematosus: Making the Case for an Expanded Psychiatric Role. JNeurol Exp Neurosci 2(1): 9-11.

Copyright: (C) 2016 Samuels et al. This is an Open Access article distributed under the terms of the Creative Commons Attribution 4.0 International License (CC-BY) (http://creativecommons. org/licenses/by/4.0/) which permits commercial use, including reproduction, adaptation, and distribution of the article provided the original author and source are credited.

Published by United Scientific Group

\begin{abstract}
Systemic Lupus Erythematous can present with psychiatric symptoms. As these symptoms are non-specific and may occur in the absence of clear systemic signs and symptoms of lupus, they are difficult to distinguish from primary psychiatric illnesses. We present the case of a young woman who, on two separate admissions, was treated for a psychiatric illness with no effect and then treated for Neuropsychiatric SLE with remarkable improvement. We discuss the atypical features that a psychiatrist can use to distinguish the two diagnoses and the role of the psychiatrist or neurologist in determining the severity of mental status changes in order to guide treatment.
\end{abstract}

\section{Introduction}

Neuropsychiatric symptoms are common manifestations of Systemic Lupus Erythematosus (SLE) with prevalence estimates ranging from $14 \%$ to $80 \%$ in adults with SLE $[1,2]$. SLE can lead to diverse psychiatric symptoms, including mood and anxiety disorders, acute confusional states, psychosis, and cognitive dysfunction [3]. Although common, Neuropsychiatric SLE (NPSLE) is arguably the least understood of all the lupus manifestations due to lack of clarity from a pathophysiologic, diagnostic, and therapeutic perspective, thereby posing multiple dilemmas in the clinical setting [4].

\section{Case Report}

Ms. Z, a 22 years-old South Asian woman with SLE and no other medical history, no past psychiatric history, no toxic habits, and no family psychiatric history presented to our hospital for one month of worsening mouth and body pain with failure to thrive. She could not give a coherent history and was resistant to medical care. On exam, Ms. $Z$ was a poorly groomed, malodorous young woman with pronounced negativism, including flat affect, poverty of speech, psychomotor slowing, and poor eye contact. She denied mood or psychotic symptoms. She was alert and could state her name and age but replied "I can't say" or "no, no, no" when asked further questions. The remainder of her neurologic exam was unremarkable. As per her family, Ms. Z lost her job and became increasingly isolative, withdrawn, and irritable approximately 9 months prior to admission. They denied depressed mood, increased energy or goal-directed activity, paranoia, and hallucinations. A review of her chart demonstrated a brief period of treatment 3 years prior to admission for SLE (met 4 out of 4 needed criteria with + ANA, + anti-dsDNA, + arthritis, + leukopenia) with low-dose prednisone and hydroxychloroquine.

Upon admission, the rheumatology service treated Ms. Z for what they described as a mild lupus flare, based upon joint findings and positive bloodwork (low c3 and c4, elevated anti-dsDNA, ESR 53, ANA 1:320). Both rheumatology 
and neurology believed her psychiatric symptoms were due to Major Depressive Disorder (MDD) and therefore did not include these symptoms in the assessment of the severity of her SLE. The initial treatment protocol consisted of low-dose IV steroids, methylprednisolone $30 \mathrm{mg}$ IV for 5 days, with the subsequent addition of hydroxychloroquine $400 \mathrm{mg}$ daily and an oral prednisone taper from $20 \mathrm{mg}$ daily down to $5 \mathrm{mg}$ daily. This medication regimen ameliorated her polyarthritis but did not improve her mental status. Ms. $\mathrm{Z}$ continued to require IV fluids and parenteral nutrition due to her extremely poor oral intake. The psychiatry consult team felt that NPSLE and other medical etiologies should be ruled out first as Ms. Z's psychiatric symptoms were non-specific and highly suggestive of delirium.

The primary medical team, in conjunction with the rheumatology and neurology consult services, initiated a complete medical and neurological work-up to rule out other causes of mental status change. This work-up included an EEG, MRI, lumbar puncture, and blood-work. CSF results were negative for signs of infection or acute inflammation. Laboratory workup revealed normal thyroid function tests, vitamin B12, folic acid, basic metabolic panel, complete blood count, and anti-TPO antibodies. HIV testing and syphilis screen were negative. EEG was normal and a CT scan of the chest, abdomen and pelvis to rule out an ovarian teratoma or a malignancy was unremarkable. Brain MRI showed nonspecific punctate white matter lesions. Two pertinent positive findings included Anti-Ribosomal $\mathrm{P}$ antibodies that were elevated at greater than 8 (normal $<1$ ) and the presence of oligoclonal bands in the CSF.

Based on these inconclusive findings, rheumatology recommended a trial of psychotropic medications as the firstline treatment for Ms. $Z$ as they remained convinced that her psychiatric symptoms were not severe and likely related to a primary psychiatric illness. The patient did not respond to trials of haloperidol up to $1 \mathrm{mg}$ total daily dose, olanzapine up to $10 \mathrm{mg}$ at bedtime, or sertraline up to $50 \mathrm{mg}$ daily. She had a marked dystonic reaction after receiving an additional $1 \mathrm{mg}$ IV haloperidol, which was given for agitation.

One month into the hospitalization, the patient's sibling visited and reported that $\mathrm{Ms}$. $\mathrm{Z}$ was confused, disoriented to time and place, and had poor verbal comprehension in her native language. This new collateral information from her sibling, in conjunction with Ms. Z's lack of response to psychotropic medication, persuaded the medical team and multiple consulting services that the patient's presentation was most consistent with an episode of delirium, likely secondary to NPSLE. At that time, a 3-day course of high-dose pulse steroids consisting of methylprednisolone $1,000 \mathrm{mg}$ daily was given to treat delirium from NPSLE. Ms. $Z$ had marked improvement in her clinical status. She was discharged one week later on prednisone $50 \mathrm{mg}$ daily and mycophenolate $500 \mathrm{mg}$ Q12 for her SLE. She was off all psychotropic medication, with better social function and intact cognition, including orientation and memory.

Approximately 3 weeks later, Ms. Z was brought back to the hospital for refusing food, social withdrawal, headache, and tearfulness. Given her increase in psychiatric symptoms off psychotropic medication and her normal blood work and physical exam, with reported good compliance to prednisone and mycophenolate, the medical services were again concerned for a primary psychiatric illness like depression. As there was no medical intervention offered, Ms. Z was transferred to the psychiatry floor for management of presumed MDD. Ms. $Z$ remained on the psychiatry floor for 3 weeks and was sequentially treated with trials of aripiprazole up to $15 \mathrm{mg}$ daily and mirtazapine $15 \mathrm{mg}$ QHS, and later olanzapine up to $20 \mathrm{mg}$ daily with concurrent lorazepam at $2 \mathrm{mg}$ Q12 for possible catatonia. Additionally, her mycophenolate was increased to $1500 \mathrm{mg} \mathrm{Q12}$ and her prednisone was lowered to $40 \mathrm{mg}$ daily. Ultimately, the inpatient psychiatric team felt there was no improvement on psychotropic medications, and they believed Ms. Z's presentation was again the result of NPSLE and not an underlying primary psychiatric disorder.

Ms. $Z$ was transferred back to the medicine service for another course of high-dose pulse steroids. This time she was treated with 1,000 mg methylprednisolone IV for 5 days. Two days into this treatment, Ms. Z exhibited improved grooming, ameliorated cognition, increased verbal fluency, and better socialization with her family. She was discharged one month after admission, on definitive SLE treatment including oral methylprednisolone and mycophenolate, and all psychotropic medications were titrated off as an outpatient. She maintained her treatment gains exclusively on immunosuppressants for $\mathrm{SLE}$ and no longer required psychiatric follow up.

\section{Discussion}

While our case is not a unique presentation of NPSLE, it highlights the difficulties of differentiating the psychiatric presentations of NPSLE from primary psychiatric conditions. NP manifestations are the presenting symptoms in up to $50 \%$ of cases of lupus and are often present without other systemic signs and symptoms of SLE [5]. There is no laboratory or radiological biomarker for establishing a diagnosis of NPSLE. MRI, EEG, and CSF findings are often absent or nonspecific and may be present in patients with SLE regardless of symptomatology $[6,7]$. The presence of anti-ribosomal $\mathrm{P}$ antibodies can point to a lupus-related psychosis but is by no means conclusive $[6,8,9]$. Thus NPSLE remains a diagnosis of exclusion [3].

Whereas excluding infections, electrolyte abnormalities, and medication effects is typically possible, excluding a primary psychiatric condition, such as major depressive disorder or schizophrenia, is more difficult as these are also diagnoses of exclusion, which affect a similar demographic as those with SLE [6]. It is important, then, to look for distinguishing clinical features of NPSLE compared with primary psychiatric conditions. In 1999, the American College of Rheumatology delineated 19 possible NP manifestations of SLE, which included psychosis, mood disorder, anxiety disorder, and cognitive disorder [3]. These categories, though, were nonspecific and no guidance was given on distinguishing them from psychiatric syndromes. It is due to this lack of specificity and low inter-rater reliability that studies of prevalence for NPSLE vary so drastically [10]. 
In the case of Ms. Z, while her symptoms might have suggested a major depressive disorder, she did not have the cardinal symptoms of depressed mood or classic anhedonia. And while her behavior could have been characterized as disorganized, she lacked definitive symptoms of schizophrenia, like delusions or hallucinations. Actually, her symptoms of mood lability and isolative, bizarre behavior did not cluster into any specific category of a primary mental illness. Additionally, Ms. $Z$ would consistently say "I can't say," which was highly suggestive of the cognitive impairment that her sibling ultimately confirmed. Lastly, Ms. Z's high sensitivity to EPS from low doses of IV haloperidol was quite atypical and has been linked to several neuropsychiatric conditions [11]. The psychiatry team favored the diagnosis of NPSLE early in both admissions because of these clinical features.

Based on our case, we suggest that the neurologist or psychiatrist should be a key member of the treatment team when negotiating the complexities of the diagnosis and treatment of suspected NPSLE. Several possible features of atypicality might be used to distinguish NPSLE from primary psychiatric syndromes. Acute onset and rapid progression, as seen during the second presentation of Ms. Z, might indicate NPSLE as most primary psychiatric disorders have an insidious onset and gradual progression. The presence of cognitive impairment, in particular alterations in attention or orientation, can indicate NPSLE and are less likely in primary psychiatric disorders. Psychiatric syndromes in DSM 5 consist of core signs or symptoms, in addition to a cluster of other manifestations, which may be lacking in NPSLE, as was the case with Ms. Z. Furthermore, as with Ms. Z, heightened sensitivity to the extrapyramidal side effects (EPS) of antipsychotics can indicate neuropsychiatric autoimmune diseases, like NPSLE [11].

Even when a diagnosis of NPSLE is heavily supported, the necessary treatment is not always clear. Treatment choices should be guided by a good assessment of the severity of a patient's NP symptoms; however, levels of severity are not clearly defined in existing literature $[3,10]$. Severe symptoms should be treated with high-dose IV steroids followed by IV cyclophosphamide as first line treatments and rituximab, IVIG, or plasmapheresis as second line treatments. When symptoms are mild to moderate, though, patients may be treated with symptomatic treatment, like antipsychotics or antidepressants, as was initially suggested with Ms. Z [10,12]. Due to a lack of clarity in the literature, and possibly due to a lack of experience in assessing psychiatric symptomatology on the part of medical and rheumatology teams, these services may not appreciate the severity of a patient's psychiatric symptoms, as was the case with Ms. Z.

\section{Conclusion}

The consultation-liaison psychiatrist or neurologist should play a crucial role in the diagnosis and management of NPSLE when it manifests with psychiatric symptoms. The psychiatrist can highlight atypical clinical features that would favor a diagnosis of NPSLE and can assess the severity of a patient's cognitive, emotional, and behavioral symptoms. An enhanced psychiatric and neurologic role could minimize prolonged morbidity, including multiple hospitalizations and exposure to psychotropic medications, for patients with severe psychiatric manifestations of NPSLE.

\section{Funding Details}

We have not received any financial support for this work.

\section{Disclosure}

The authors have no conflicts of interest or any sources of funding to report. We certify that the submission is original work and is not under review at any other publication.

\section{References}

1. Hanly JG, McCurdy G, Fougere L, Douglas JA, Thompson K. 2004. Neuropsychiatric events in systemic lupus erythematosus: attribution and clinical significance. J Rheumatol 31(11): 2156-2162.

2. Stojanovich L, Zandman-Goddard G, Pavlovich S, Sikanich N. 2007. Psychiatric manifestations in systemic lupus erythematosus. Autoimmunity Reviews 6(6): 421-426. doi: 10.1016/j.autrev.2007.02.007

3. 1999. The American College of Rheumatology nomenclature and case definitions for neuropsychiatric lupus syndromes. Arthritis Rheum 42(4): 599-608. doi: 10.1002/1529-0131(199904)42:4<599::AIDANR2>3.0.CO;2-F

4. Calabrese LV, Stern TA. 1995. Neuropsychiatric manifestations of systemic lupus erythematosus. Psychosomatics 36(4): 344-359. doi: 10.1016/S0033-3182(95)71644-9

5. Kovacs JA, Urowitz MB, Gladman DD. 1993. Dilemmas in neuropsychiatric lupus. Rheum Dis Clin North Am 19(4): 795-814.

6. Sibbitt WL Jr, Brooks WM, Kornfeld M, Hart BL, Bankhurst AD, et al. 2010. Magnetic resonance imaging and brain histopathology in neuropsychiatric systemic lupus erythematosus. Semin Arthritis Rheum 40(1): 32-52. doi: 10.1016/j.semarthrit.2009.08.005

7. Abreu MR, Jakosky A, Folgerini M, Brenol JC, Xavier RM, et al. 2005. Neuropsychiatric systemic lupus erythematosus: correlation of brain MR imaging, CT, and SPECT. Clin Imaging 29(3): 215-221. doi: 10.1016/j.clinimag.2004.07.007

8. Postal M, Costallat LT, Appenzeller S. 2011. Neuropsychiatric manifestations in systemic lupus erythematosus: epidemiology, pathophysiology and management. CNS Drugs 25(9): 721-736. doi: 10.2165/11591670-000000000-00000

9. Popescu A, Kao AH. 2011. Neuropsychiatric systemic lupus erythematosus. Curr Neuropharmacol 9(3): 449-457. doi: 10.2174/157015911796557984

10. Magro-Checa C, Zirkzee EJ, Huizinga TW, Steup-Beekman GM. 2016. Management of neuropsychiatric systemic lupus erythematosus: current approaches and future perspectives. Drugs 76(4): 459-483. doi: 10.1007/s40265-015-0534-3

11. Bergink V, Armangue T, Titulaer MJ, Markx S, Dalmau J, et al. 2015. Autoimmune encephalitis in postpartum psychosis. Am J Psychiatry 172(9): 901-908. doi: 10.1176/appi.ajp.2015.14101332

12. Stojanovic L, Stojanovich R, Kostich V, Dzjolich E. 2003. Neuropsychiatric lupus favourable response to low dose i.v. cyclophosphamide and prednisolone (pilot study). Lupus 12(1): 3-7. doi: 10.1191/09612033031u251oa 